\title{
Occult hemoptysis: a rare case of esthesioneuroblastoma
}

\author{
Shalini Jain*, Kanwer Sen \\ Department of ENT and Head and Neck Surgery, Atal Bihari Vajpayee Institute of Medical Sciences and Dr. Ram \\ Manohar Lohia Hospital, New Delhi, India
}

Received: 09 May 2020

Revised: 17 June 2020

Accepted: 22 June 2020

\section{*Correspondence:}

Dr. Shalini Jain,

E-mail: drshalini0201@gmail.com

Copyright: () the author(s), publisher and licensee Medip Academy. This is an open-access article distributed under the terms of the Creative Commons Attribution Non-Commercial License, which permits unrestricted non-commercial use, distribution, and reproduction in any medium, provided the original work is properly cited.

\begin{abstract}
Esthesioneuroblastoma forms a part of the spectrum of rare malignancies of the olfactory neuroepitheliem. The rarity of the tumour precludes systematic approach to clinical diagnosis and management. This study involves a case of 68 years old female who presents with chief complaints of hemoptysis for 3 months in the medical outpatient department with no nasal signs and symptoms. After referral to chest physician she is referred for ear nose and throat opinion. Esthesioneuroblastoma presentation is very varied and the case reports published have been very few. In view of the rarity of the tumour, clinical suspicion is essential in unusual presentation of hemoptysis.
\end{abstract}

Keywords: Esthesioneuroblastoma, Olfactory neuroblastoma, Malignant tumour of nasal cavity

\section{INTRODUCTION}

Where the bleeding coming from is hemoptysis is not an uncommon referral and it can be spurious, occult, hidden or factitious. The commonly found site and cause of bleeding in ENT. ${ }^{1}$ Scenario includes gum (gingivitis), upper and lower respiratory tract infections, malignancies, bleeding disorders, anticoagulants or from nose and oral cavity. For the elusive diagnosis, we need to locate the cause and investigate for upper airway etiology. ${ }^{2}$

\section{CASE REPORT}

Here is a case of 68 years old female, resident of Begusarai Bihar who presented to the medical outpatient department with the chief complaints of blood-stained sputum for 3 months, bright red in colour. There was no history of hypertension, diabetes mellitus, tuberculosis, or drug allergy. Systemic examination was clinically normal. Patient was sent for respiratory physician opinion and the underwent flexible bronchoscopy, contrast enhanced computed tomography of the chest and
Montoux test. These tests were reported to be within normal limits. After this patient was sent for ear, nose and throat consultation where we did nasal endoscopy and found there was streaking of blood on the left nasal wall, but no mass was visualized. Hopkins laryngeal examination revealed mild left vocal cord oedema and congestion; and on computed tomogram of nose and paranasal sinuses, (Figure 1), there was left maxillary polyp suggestive of sinusitis. Patient was advised for endoscopic middle meatal antrostomy with biopsy. Preoperatively, left concha bullosa with left hemorrhagic maxillary polyp was found. Uncinectomy was done and the polyp was removed in toto and sent for histopathological examination. Remaining maxillary sinus mucosa was healthy. Histopathological report came to be chronic non-specific inflammation. Presently patient improved symptomatically and was kept in regular follow up. After a month, there was recurrence of blood-stained sputum. Nasal endoscopy was normal, laryngeal examination revealed smooth reddish polypoidal mass on the superior surface and free margin of left true vocal cord, both vocal cords mobile (Figure 2). Having failed to locate source of bleed and a polypoid 
mass on the vocal cord and persistence of symptoms, patient underwent micro laryngeal examination with biopsy, and histopathological report came to be benign laryngeal nodules. Patient was asked for regular follow up.

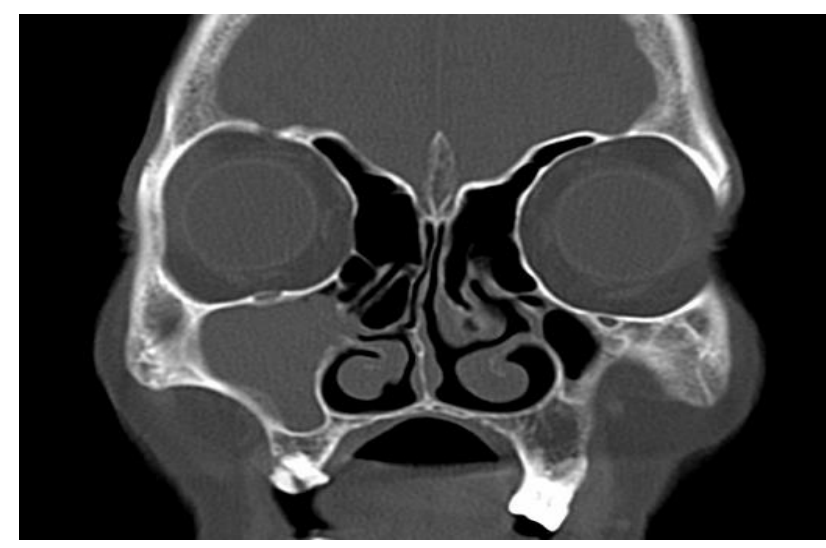

Figure 1: Pre-operative computed tomogram of nose and paranasal sinuses suggestive of maxillary polyp sinusitis.

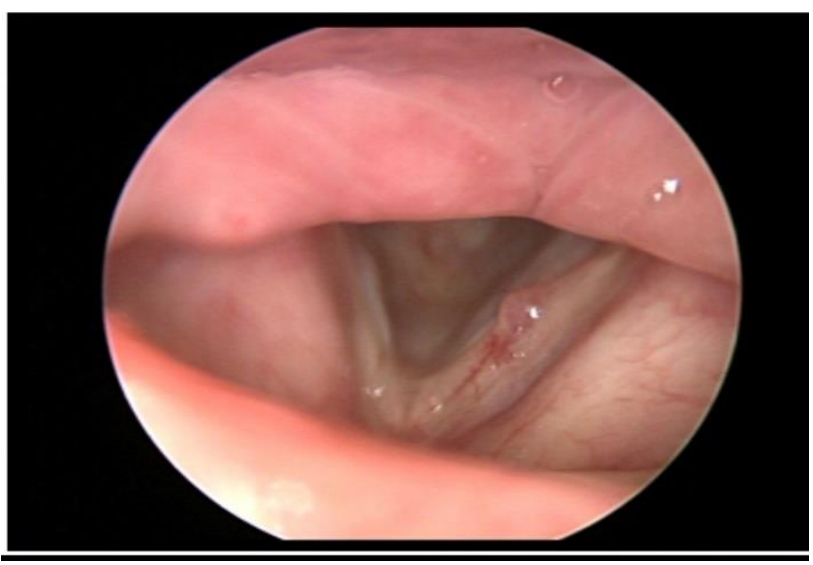

Figure 2: 90 degrees laryngoscopy showing smooth reddish polypoidal mass on the superior and free surface of left vocal cord.

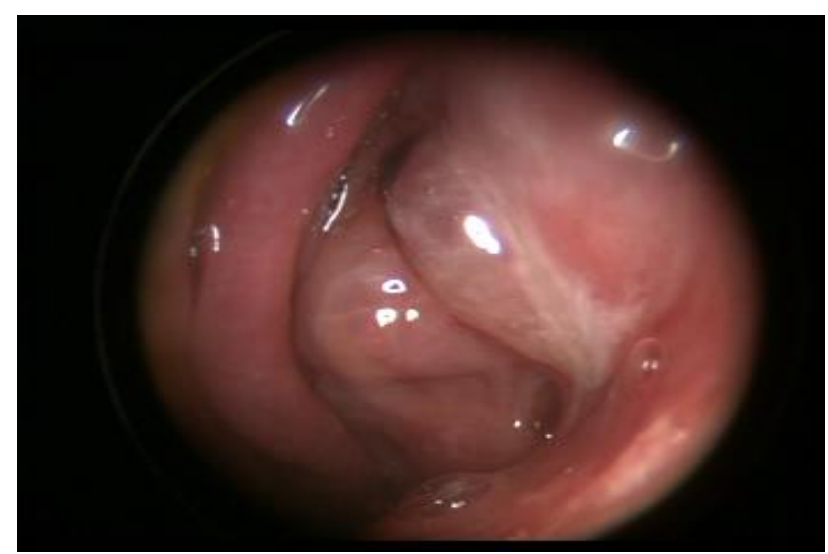

Figure 3: Pre-operative nasal endoscopy showing blood stained pulsatile discharge near left ostia with granulations.
After a month again there was recurrence of hemoptysis and this time there was epistaxis as well. Nasal endoscopy revealed blood stained pulsatile discharge near left maxillary ostia with granulations (Figure 3).

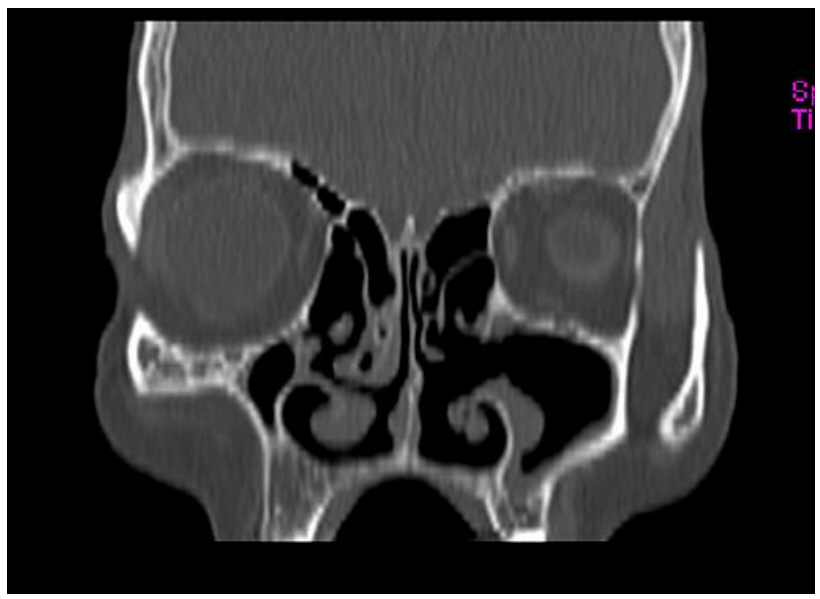

Figure 4: Pre-operative contrast enhanced computed tomogram showing mild enhancing soft tissue mass in left maxillary antrum. No evidence of bony destruction.

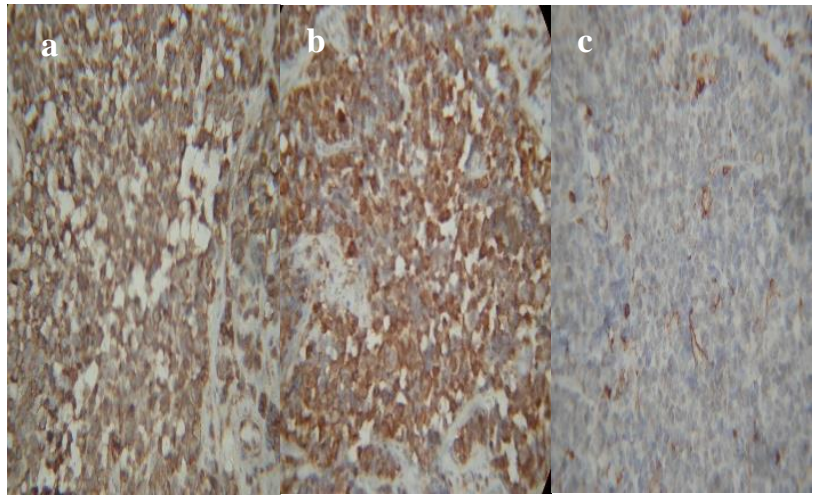

Figure 5: (a) Immunohistochemical (IHC) staining positivity with chromogranin, (b) IHC staining neuron specific enolase positive and (c) IHC staining human leukocyte common antigen (LCA) negative.

Repeat computed tomogram of nose and paranasal sinuses revealed mild enhancing soft tissue mass in left maxillary antrum, with no evidence of bony destruction (Figure 4).

Patient was planned for lateral rhinotomy with medial maxillectomy for tissue biopsy and to address the lesion on the medial aspect of the maxillary sinus. Histopathological examination showed poorly differentiated carcinoma with chronic non-specific inflammation, and IHC reports Figure 5 (a-c), were suggestive of fragments of respiratory epithelium with squamous metaplasia, with tumour cells arranged in nests and clusters. Cells have distinct moulded pattern with salt and pepper chromatin. Mitosis is brisk, no necrosis, tumour is invading bone. 
Results of IHC staining- chromogranin: positive; synaptophysin: positive; neuron specific enolase: positive; S-100 protein: positive; cytokeratin: positive. It was further decided to send the patient for post-operative radiotherapy.

Table 1: Modified Kadish classification.

\begin{tabular}{|lll|}
\hline Stage & Description & $\begin{array}{l}\text { 5 years } \\
\text { survival }\end{array}$ \\
\hline A & Tumor confined to nasal cavity & $75-91$ \\
\hline B & $\begin{array}{l}\text { Nasal cavity and paranasal } \\
\text { sinuses }\end{array}$ & $68-71$ \\
\hline C & $\begin{array}{l}\text { Tumor extends beyond nasal } \\
\text { cavity and paranasal sinuses, } \\
\text { including skull base, orbit or } \\
\text { cribriform plate }\end{array}$ & $41-47$ \\
\hline D & $\begin{array}{l}\text { Tumor metastasizes to cervical } \\
\text { lymph nodes and beyond }\end{array}$ & $<40$ \\
\hline
\end{tabular}

\section{DISCUSSION}

Neuroendocrine tumours are tumours of the interface between the endocrine (hormonal) system and the nervous system.

The types are carcinoids - 60\%; out of which $90 \%$ are non-functioning, pancreatic - 90\%; $80 \%$ functioning and rare sites include mainly lungs.

In the paranasal sinuses these are very rare. A 1997 literature search identified 1,457 cases in the published literature. ${ }^{3}$ It arises from the olfactory neuroepithelium of the roof of the nasal cavity and paranasal sinuses. It was first described by Berger and Luc and is also known as esthesioneuroblastoma, esthesioneurocytoma and neuroendocrine carcinoma. ${ }^{4,5}$ It represents 3 percent of sino nasal malignancy, with no sex predilection. It has a bimodal age distribution peaking at 15 years and 50 years of age. Our patient was a 68 years old woman. Esthesioneuroblastoma is a slow growing tumour and the patients may have history of progressive symptomatology for months to years.

\section{Clinical features}

The symptoms of esthesioneuroblastoma include nasal obstruction, rhinorrhoea, epistaxis, facial pain and diplopia. However, patients more often present with nonspecific symptoms like our patient which presented with occult hemoptysis, and such cases are diagnosed late with advanced disease. ${ }^{6-8}$ The staging system presented by Kadish et al in 1976 and modified by Morita et al in 1993 is based upon predictive disease related mortality. ${ }^{7,8}$ Two other staging methods, the bitter method and the Dulguerov method have also been described and used.

Esthesioneuroblastoma has a locally invasive behavior. It spreads through the submucosa to the surrounding structures and involves anterior cranial vault destroying the cribriform plate with propagation along the perineural spaces of olfactory nerves. Orbital extension takes place through the erosion of lamina papyracea. It has been reported that upto 70 percent of patients present at a locally advanced stage. ${ }^{6-8}$

\section{Investigations}

Nasal endoscopy usually confirms an exophytic or sessile mass arising from the superior portion of the olfactory mucosa. High resolution computed tomography scan and magnetic resonance imaging can be used as supportive investigation to accurately delineate the extent of the tumour into surrounding structures such as cribriform plate, fovea ethmoidalis, anterior cranial fossa and retro maxillary space.

\section{Histological features}

The hallmark of esthesioneuroblastoma is the formation of tumour cells into rosettes, pseudo rosettes or sheets and clusters separated by a fibrovascular stroma. Necrosis is rare, mitosis is brisk, with mild to moderate nuclear pleomorphism. Electron microscopy and IHC studies are needed to establish the diagnosis. Hyam's et al introduced historical grading system (Table 2). ${ }^{9}$

Table 2: Hyam's histopathological grades for esthesioneuroblastoma.

\begin{tabular}{|lllllll|}
\hline Grade & $\begin{array}{l}\text { Lobular architecture } \\
\text { preservation }\end{array}$ & Mitotic index & $\begin{array}{l}\text { Nuclear } \\
\text { polymorphism }\end{array}$ & $\begin{array}{l}\text { Fibrillary } \\
\text { matrix }\end{array}$ & Rosettes & Necrosis \\
\hline I & + & None & None & Prominent & $\begin{array}{l}\text { Homer wright } \\
\text { (HW) rosettes }\end{array}$ & None \\
\hline II & + & Low & Moderate & Present & HW rosettes & None \\
\hline III & $+/-$ & Moderate & Prominent & Low & $\begin{array}{l}\text { Flexner- } \\
\text { wintersteiner } \\
\text { rosettes }\end{array}$ & Rare \\
\hline IV & $+/-$ & High & Marked & Absent & None & Frequent \\
\hline
\end{tabular}




\section{CONCLUSION}

There is no universally accepted optimal treatment for esthesioneuroblastoma in view of its rarity and the lack of randomized trials. Small tumours confined to the nasal or paranasal sinuses may be considered for an endoscopic resection provided appropriate expertise is available. However, a combined treatment modality approach with surgery and radiotherapy is needed for more advanced stage disease like in our patient. Craniofacial resection is the standard surgical approach for most cases enabling en bloc resection with the cribriform plate. This has been shown to improve overall as well as disease free survival. Five-year survival has been shown to improve from 37.5 to 82 percent with craniofacial resection. However, despite macroscopic clearance, there is a relatively high risk of locoregional recurrence of 10-30 percent and therefore adjuvant therapies with radiotherapy with or without chemotherapy is required.

Funding: No funding sources

Conflict of interest: None declared

Ethical approval: Not required

\section{REFERENCES}

1. Bannister M, See AKW. Evidenced-based management of hemoptysis by otolaryngologists. J Laryngol Otol. 2015;129(8):807-11.
2. Satiago S, Tobias J, Williams AJ. A reappraisal of the causes of hemoptysis. Arch Int Med. 1991;151:2249-451.

3. Broic G, Pagliari A, Ottaviani F. Esthesioneuroblastoma: a general review of the cases published since the discovery of the tumour in 1924. Anticancer Res. 1997;17(4A):2683-706.

4. Berger L, Luc R. Esthesioneuroblastoma olfactif. Bull Assoc Fr Etud Cancer. 1924;3:410-21.

5. Elkon D, Hightower SI, Lim ML. Esthesioneuroblastoma. Cancer. 1979;44:1087-94.

6. Levine PA, Gallagher R, Coantrell RW. Esthesioneuroblastoma; reflections of a 21 years' experience. Laryngoscope. 1999;109:1539-43.

7. Kadish S, Goodman M, Wang CC. Olfactory neuroblastoma: a clinical analysis of 17 cases. Cancer. 1976;37:1571-6.

8. Morita A, Ebersold MJ, Olsen KD. Esthesioneuroblastoma: prognosis and management. Neurosurgery. 1993;32:706-14.

9. Stell and Maran Textbook of Head and Neck surgery and oncology fifth edition. 2012;41:792-5.

Cite this article as: Jain S, Sen K. Occult hemoptysis: a rare case of esthesioneuroblastoma. Int J Otorhinolaryngol Head Neck Surg 2020;6:1542-5. 